International Journal of Computational and Applied Mathematics.

ISSN 1819-4966 Volume 13, Number 1 (2018), pp. 33-41

(C) Research India Publications

https://dx.doi.org/10.37622/IJCAM/13.1.2018.33-41

\title{
Generalized biderivations on prime and semiprime rings
}

\author{
Asma Ali, Faiza Shujat and Kapil Kumar \\ Department of Mathematics, \\ Aligarh Muslim University, Aligarh-202002 (India).
}

\begin{abstract}
The purpose of our paper is to prove some results concerning symmetric generalized biderivation on prime (semiprime) rings, which are of independent interest. Moreover, we generalizes the results of Argac [1, Theorem 3.1, Theorem 3.5].
\end{abstract}

AMS subject classification: 16W20 16N30 16R40.

Keywords: Prime rings, semiprime rings, symmetric generalized biderivation.

\section{Introduction}

Throughout this paper $R$ will represent an associative ring with center $Z(R)$. A ring $R$ is said to be prime (resp. semiprime) if $a R b=\{0\}$ implies that either $a=0$ or $b=0$ (resp. $a R a=0$ implies that $a=0$ ). We shall write $[x, y]$ the commutator $x y-y x$. We make extensive use of basic commutator identities $[x y, z]=[x, z] y+x[y, z]$ and $[x, y z]=[x, y] z+y[x, z]$. An additive mapping $d: R \longrightarrow R$ is said to be a derivation if $d(x y)=d(x) y+x d(y)$, for all $x, y \in R$. A derivation $d$ is inner if there exists an element $a \in R$ such that $d(x)=[a, x]$, for all $x \in R$. Gy. Maksa [2] introduced the concept of a symmetric biderivation (see also [3], where an example can be found). It was shown in [2] that symmetric biderivations are related to general solution of some functional equations. Some results on symmetric biderivation in prime and semiprime rings can be found in [7], [8] and [9]. The notion of commuting mappings is closely connected with the notion of biderivations. Every commuting mapping $f: R \longrightarrow R$ gives rise to a biderivation on $R$. Namely linearizing $[x, f(x)]=0$, for all $x, y \in R$, $(x, y) \longrightarrow[f(x), y]$ is a biderivation (moreover, all derivations appearing are inner). There has been ongoing interest concerning the relationship between the commutativity of a ring and the existence of certain specific types of derivations. 
Amapping $D(.,):. R \times R \longrightarrow R$ is said to be symmetric if $D(x, y)=D(y, x)$, for all $x, y \in R$. A mapping $f: R \longrightarrow R$ defined by $f(x)=D(x, x)$, where $D(.,$.$) :$ $R \times R \longrightarrow R$ is a symmetric mapping, is called the trace of $D$. It is obvious that in the case $D(.,):. R \times R \longrightarrow R$ is a symmetric mapping which is also biadditive (i.e. additive in both arguments), the trace $f$ of $D$ satisfies the relation $f(x+y)=f(x)+f(y)+$ $2 D(x, y)$, for all $x, y \in R$. A biadditive symmetric mapping $D(.,):. R \times R \longrightarrow R$ is called a symmetric biderivation if $D(x y, z)=D(x, z) y+x D(y, z)$, for all $x, y, z \in R$. Obviously, in this case the relation $D(x, y z)=D(x, y) z+y D(x, z)$ is also satisfied for all $x, y, z \in R$. The notion of generalized symmetric biderivations introduced by Nurcan [1], which is defined as follows:

Let $R$ be a ring and $D(.,):. R \times R \longrightarrow R$ be a biadditive map. A biadditive mapping $\Delta: R \times R \longrightarrow R$ is said to be generalized biderivation if for every $x \in R$, the map $y \longrightarrow \Delta(x, y)$ is a generalized derivation of $R$ associated with function $y \longrightarrow D(x, y)$ as well as if for every $y \in R$, the map $x \longrightarrow \Delta(x, y)$ is a generalized derivation of $R$ associated with function $x \longrightarrow D(x, y)$, for all $x, y \in R$. It also satisfies $\Delta(x, y z)=\Delta(x, y) z+y D(x, z)$ and $\Delta(x y, z)=\Delta(x, z) y+x D(y, z)$, for all $x, y, z \in$ $R$. The trace $g$ of $\Delta$ is defined as $\Delta(x, x)=g(x)$, which satisfies $g(x+y)=$ $g(x)+g(y)+2 \Delta(x, y)$, for all $x, y \in R$.

\section{Main Result}

We shall frequently use the following identities and several well known fact about the prime ring without specific mention.

$$
\begin{gathered}
{[x y, z]=x[y, z]+[x, z] y} \\
{[x, y z]=y[x, z]+[x, y] z} \\
x \circ y z=(x \circ y) z-y[x, z]=y(x \circ z)+[x, y] z \\
(x y) \circ z=x(y \circ z)-[x, z] y=(x \circ z) y+x[y, z]
\end{gathered}
$$

Lemma 2.1. [4] Suppose that

$$
\sum_{i=1}^{n} f_{i}(z) x a_{i}+\sum_{j=1}^{n} c_{j} z h_{j}(x)=0 \text { for all } x, z \in R,
$$

where $a_{i}, c_{j} \in R, f_{i}: R \longrightarrow R$ and $h_{j}: R \longrightarrow R C$ are any maps. If the sets $\left\{a_{1}, a_{2}, \ldots\right\}$ and $\left\{c_{1}, c_{2}, \ldots\right\}$ are $\mathrm{C}$-independent, then there exists $q_{i j} \in Q(S=R C)$ for $i=1,2, \ldots, k$ and $j=1,2, \ldots, n$ such that $f_{i}(z)=-\sum_{j=1}^{n} c_{j} z q_{i j}, h_{j}(x)=$ $\sum_{i=1}^{n} q_{i j} x a_{i}$ for all $x, z \in R$ 
Lemma 2.2. [4] If $a_{i}, b_{i} \in Q(S)$ satisfy $\sum_{i=1}^{n} a_{i} x b_{i}=0$ for all $x \in R$, then $a_{i}^{\prime} s$ and $b_{i}^{\prime} s$ are C-dependent, unless $a_{i}=0$ or all $b_{i}=0$.

Lemma 2.3. [3] If $q \in Q(S)$ and $q$ centralizes $R$, then $q \in C$.

Theorem 2.4. Let $R$ be a 2-torsion free prime ring and $\Delta$ be symmetric generalized biderivation of $R$ with associated biderivation $D$. If $\Delta([x, y],[u, v])=0$ for all $x, y, u, v \in$ $R$, then either $R$ is commutative or $\Delta$ acts as a bimultiplier.

Proof. We have

$$
\Delta([x, y],[u, v])=0 \text { for all } x, y, u, v \in R .
$$

Replacing $y$ by $y x$ in (1), and use (1) to get

$$
[x, y] D(x,[u, v])=0 \text { for all } x, y, u, v \in R .
$$

Replacing $u$ by $u v$ in (2), we have

$$
[x, y][u, v] D(x, v)=0 \text { for all } x, y, u, v \in R .
$$

Substitute $r y$ for $y$ in (3) to find

$$
[x, y] y[u, v] D(x, v)=0 \text { for all } x, y, u, v, \in R .
$$

This implies that $[x, r] D(w, z) y[u, v] D(x, v)=0$ for all $x, y, u, v, w \in R$. Since $R$ is prime, we have $[x, r] D(w, z)=0$ for all $x, r, w, z \in R$. Replacing $r$ by $r s$, we obtain

$$
[x, r] s D(w, z)=0 \text { for all } x, r, w, z, s \in R .
$$

Using primeness of $R$, we have either $[x, r]=0$ or $D(w, z)=0$ for all $x, r, w, z \in R$. If $[x, r]=0$, then $R$ is commutative. In the later case we get $\Delta$ acts as a bimultiplier.

Corollary 2.5. Let $R$ be a 2-torsion free prime ring and $\Delta$ be symmetric generalized biderivation of $R$ with associated biderivation $D$. If $\Delta([x, y], z)=0$ for all $x, y, z \in R$, then either $R$ is commutative or $\Delta$ acts as a bimultiplier.

Theorem 2.6. Let $R$ be a 2-torsion free prime ring and $\Delta$ be symmetric generalized biderivation of $R$ with associated biderivation $D$. If $\Delta([x, y], u \circ v)=0$ for all $x, y, u, v \in$ $R$, then either $R$ is commutative or $\Delta$ acts as a bimultiplier.

Proof. Suppose that

$$
\Delta([x, y], u \circ v)=0 \text { for all } x, y, u, v \in R
$$

Replacing $y$ by $y x$, in (6), we get

$$
\Delta([x, y], u \circ v) x+[x, y] D(x, u \circ v)=0 \text { for all } x, y, u, v \in R
$$


Now using (6), we obtain

$$
[x, y] D(x, u \circ v)=0 \text { for all } x, y, u, v \in R .
$$

Again replacing $u$ by $u v$ in (8), we have

$$
[x, y] D(x, u \circ v) v+[x, y](u \circ v) D(x, v)=0 \text { for all } x, y, u, v \in R .
$$

By equation (8), we get

$$
[x, y](u \circ v) D(x, v)=0 \text { for all } x, y, u, v \in R .
$$

Now substituting $y r$ instead of $y$ in (9) and using (9), we find that

$$
[x, y] r(u \circ v) D(x, v)=0 \text { for all } x, y, u, v \in R .
$$

Replacing $r$ by $D(w, t) r$ in (10), we have

$$
[x, y] D(w, t) r(u \circ v) D(x, v)=0 \text { for all } x, y, u, v, w, t \in R .
$$

Using primeness of $R$, we have either $[x, y] D(w, t)=0$ or $(u \circ v) D(x, v)=0$. Now, first suppose that

$$
[x, y] D(w, t)=0 \text { for all } x, y, w, t \in R \text {. }
$$

Substituting $y s$ instead of $y$ in (11) and using (11), we have

$$
[x, y] s D(w, t)=0 \text { for all } x, y, w, t \in R .
$$

As $R$ is prime, then either $[x, y]=0$ or $D(w, t)=0$. If $[x, y]=0$, then $R$ is commutative. In the later case $\Delta$ acts as a bimultiplier. In second case suppose

$$
(u \circ v) D(x, v)=0 \text { for all } x, u, v \in R .
$$

Substituting $(u \circ v) t$ instead of $u \circ v$, we obtain

$$
(u \circ v) t D(x, v)=0 \text { for all } x, u, v, t \in R .
$$

Using primeness of $R$, either $u \circ v=0$ or $D(x, v)=0$. If $u \circ v=0$, then $\mathrm{R}$ is commutative. In the later case we get $\Delta$ acts as a bimultiplier.

Theorem 2.7. Let $R$ be a 2-torsion free prime ring and $\Delta$ be symmetric generalized biderivation of $R$ with associated biderivation $D$. If $\Delta(x \circ y, u \circ v)=0$ for all $x, y, u, v \in$ $R$, then either $R$ is commutative or $\Delta$ acts as a bimultiplier.

Proof. Suppose that

$$
\Delta(x \circ y, u \circ v)=0 \text { for all } x, y, u, v \in R .
$$


Replacing $y x$ instead of $y$ in (14), we get

$$
\Delta(x \circ y, u \circ v) x+(x \circ y) D(x, u \circ v)=0 \text { for all } x, y, u, v \in R .
$$

Now using (14), we obtain

$$
(x \circ y) D(x, u \circ v)=0 \text {, for all } x, y, u, v \in R .
$$

Again replacing $u v$ instead of $u$ in (15), we have

$$
(x \circ y)(D(x,(u \circ v) v+(u \circ v) D(x, v))=0 \text { for all } x, y, u, v \in R .
$$

This implies that

$$
(x \circ y) D(x,(u \circ v) v+(x \circ y)(u \circ v) D(x, v)=0 \text { for all } x, y, u, v \in R .
$$

By equation (15), we get

$$
(x \circ y)(u \circ v) D(x, v)=0 \text { for all } x, y, u, v \in R .
$$

Replacing $y r$ for $y$ and using (16), we get

$$
[x, y] r(u \circ v) D(x, v)=0 \text { for all } x, y, u, v, r \in R .
$$

Again substituting $D(w, z) r$ instead of $r$, we have

$$
[x, y] D(w, z) r(u \circ v) D(x, v)=0 \text { for all } x, y, u, v, t \in R .
$$

This implies that

$$
[x, y] D(w, z) R(u \circ v) D(x, v)=0 \text { for all } x, y, u, v, t \in R .
$$

Using primeness of $R$, we have either $[x, y] D(w, z)=0$ or $(u \circ v) D(x, v)=0$ for all $x, y, u, v, z, w \in R$. First suppose

$$
[x, y] D(w, z)=0 .
$$

Now we replace $y$ by $y s$, then we have $[x, y] s D(w, z)=0$. Since $R$ is prime then we have either $[x, y]=0$ or $D(w, z)=0$. If $[x, y]=0$ then $R$ is commutative. In the later case $\Delta$ acts as bimultiplier. Similarly, we consider second case $(u \circ v) D(x, v)=0$. Now replacing $(u \circ v) r$ instead of $(u \circ v)$, we obtain

$$
(u \circ v) r D(x, v)=0 .
$$

Using primeness of $R$, we have, either $(u \circ v)=0$ or $D(x, v)=0$. If $(u \circ v)=0$ then $R$ is commutative. In the later case we get $\Delta$ acts as a bimultiplier.

Corollary 2.8. Let $R$ be a 2-torsion free prime ring and $\Delta$ be symmetric generalized biderivation of $R$ with associated biderivation $D$. If $\Delta(x \circ y, z)=0$ for all $x, y, z \in R$. then either $R$ is commutative or $\Delta$ acts as a bimultiplier.

Theorem 2.9. Let $R$ be a 2 -torsion free prime ring and $\Delta_{1}, \Delta_{2}$ be symmetric generalized biderivations of $R$ with associated biderivations $D_{1}, D_{2}$ respectively. If $a \Delta_{1}(x, x)=$ $\Delta_{2}(x, x) a$ for all $x \in R$, then one of the following holds: 
1. $a \in C$ then either $a=0$ or $\Delta_{1}=\Delta_{2}$.

2. There exist $p, q \in Q(S)$ such that $a \Delta_{1}(x, y)=x p a-\operatorname{axp}, \Delta_{2}(x, y)=[x, p]$, $D_{1}(r, y)=\lambda[r, a], D_{2}(r, y)=[r, p]$.

Proof. We have

$$
a \Delta_{1}(x, x)=\Delta_{2}(x, x) a, \text { for all } x \in R .
$$

Linearizing (18) and using the symmetry, we find

$$
a \Delta_{1}(x, y)=\Delta_{2}(x, y) a, \text { for all } x, y \in R .
$$

Substitute $x r$ for $x$ in (19) to get

$$
a \Delta_{1}(x, y) r+a x D_{1}(r, y)-\Delta_{2}(x, y) r a-x D_{2}(r, y) a=0 \text {, for all } x, y, r \in R \text {. }
$$

If setting in (20), keeping $y$ as a constant

$\delta_{1}=a \Delta_{1}(x, y), \delta_{2}=-\Delta_{2}(x, y), \beta_{1}(r)=D_{1}(r, y)$ and $\beta_{2}(r)=-D_{2}(r, y) a$. Then (20) can be written as

$$
\sum_{i=1}^{2} \delta_{i}(x) r a_{i}+\sum_{j=1}^{2} c_{j} x \beta_{j}(r)=0 \text { for all } x, r \in R
$$

where $a_{1}=1, a_{2}=a, c_{1}=a, c_{2}=1$. Hence we have the following possibilities:

1. if $a \in C$ it implies that either $a=0$ or $\Delta_{1}=\Delta_{2}$.

2. if $a \notin C$, then $\{1, a\}$ are $C$-independent. Hence by Lemma 2.1, there exists an element $q_{i j} \in Q(S), i, j=1,2$ such that $\delta_{j}(x)=-\sum_{i=1}^{2} c_{i} x q_{i j}, \beta_{i}(r)=$ $\sum_{j=1}^{2} q_{i j} r a_{j}$ for all $x, r \in R$. Then we have for all $x, y, r \in R$

$$
\begin{gathered}
a \Delta_{1}(x, y)=-x q_{21}-a x q_{11} . \\
\Delta_{2}(x, y)=a x q_{12}+x q_{22} . \\
D_{1}(r, y)=q_{11} r+q_{12} r a . \\
-D_{2}(r, y) a=q_{21} r+q_{22} r a .
\end{gathered}
$$


Replace $x$ by $x u$ in (22) and using (24) we get

$$
x\left(\left[u, q_{21}\right]\right)+a x\left(u q_{11}+q_{12} u a\right)=0 .
$$

We conclude from (26) that

$$
q_{21} \in C \text { for all } u \in R,
$$

and $\left(u q_{11}+q_{12} u a\right)=0$. As $\{1, a\}$ are $C$-independent. Using Lemma 2.3 and Lemma 2.2, we arrive at

$$
q_{12} \in C
$$

and

$$
q_{12} a=-q_{11}
$$

Substitute $x r$ for $x$ in (23), we find

$$
\Delta_{2}(x r, y)=\operatorname{axr}_{12}+x r q_{22} \text { for all } x, y, r \in R .
$$

This gives that

$$
\Delta_{2}(x, y) r+x D_{2}(r, y)=\operatorname{axrq}_{12}+x r q_{22} \text { for all } x, y, r \in R .
$$

By (24), we get

$$
\left(a x q_{12}+x q_{22}\right) r+x D_{2}(r, y)=\operatorname{axr} q_{12}+x r q_{22} \text { for all } x, y, r \in R .
$$

Now using (28), we obtain

$$
x\left(D_{2}(r, y)+\left[q_{22}, r\right]\right)=0 \text { for all } r, x, y \in R .
$$

Since $R$ is prime, we have

$$
D_{2}(r, y)=\left[r, q_{22}\right] \text { for all } r, x, y \in R .
$$

Now comparing (25) and (30), we obtain

$$
q_{21}=-q_{22} a \text {. }
$$

From (24) and (29), we have $D_{1}(r, y)=q_{12}([r, a])$. Now, combining (23) and (29), we get

$$
\Delta_{2}(x, y)=x q_{22}-q_{11} x \text {. }
$$

Similarly, we are combining (22) and (32), we get

$$
a \Delta_{1}(x, y)=x q_{22} a-a x q_{11} .
$$


If we set $q_{12}=\lambda, q_{11}=q_{22}=p$ and for all $r, x, y \in R$, then we have the following form:

$$
\begin{gathered}
a \Delta_{1}(x, y)=x p a-a x p . \\
\Delta_{2}(x, y)=[x, p] . \\
D_{1}(r, y)=\lambda[r, a] . \\
D_{2}(r, y)=[r, p] .
\end{gathered}
$$

Corollary 2.10. Let $R$ be a 2-torsion free prime ring and $\Delta$ be symmetric generalized biderivation of $R$ with associated biderivation $D$. If $\Delta([x, y],[u, v])=0$ for all $x, y$, $u, v \in I$. then either $\Delta$ acts as a bimultiplier or $[I, I] I=0$, where $I$ is a left ideal of ring $R$.

The following example illustrates that $R$ to be prime is essential in the hypothesis of the above results.

Example 2.11. Let $R=\left\{\left(\begin{array}{lll}0 & a & b \\ 0 & 0 & c \\ 0 & 0 & 0\end{array}\right) \mid a, b, c \in \mathbb{Z}\right.$, ring of integers $\}$. Define maps $\Delta, D: R \times R \longrightarrow R$ by

$$
D\left(\left(\begin{array}{ccc}
0 & a_{1} & b_{1} \\
0 & 0 & c_{1} \\
0 & 0 & 0
\end{array}\right),\left(\begin{array}{ccc}
0 & a_{2} & b_{2} \\
0 & 0 & c_{2} \\
0 & 0 & 0
\end{array}\right)\right)=\left(\begin{array}{ccc}
0 & 0 & 0 \\
0 & 0 & 0 \\
0 & 0 & 0
\end{array}\right)
$$

and

$$
\Delta\left(\left(\begin{array}{ccc}
0 & a_{1} & b_{1} \\
0 & 0 & c_{1} \\
0 & 0 & 0
\end{array}\right),\left(\begin{array}{ccc}
0 & a_{2} & b_{2} \\
0 & 0 & c_{2} \\
0 & 0 & 0
\end{array}\right)\right)=\left(\begin{array}{ccc}
0 & a_{1} a_{2} & 0 \\
0 & 0 & 0 \\
0 & 0 & 0
\end{array}\right)
$$

Then $\Delta$ is a generalized biderivation associated with a biderivation $D$ satisfying hypothesis of the Theorems 2.1, 2.3, 2.4 and Corollaries 2.2 and 2.5. However, $R$ is not commutative.

\section{References}

[1] Argac, N., Generalized derivations of prime rings, Algebra Colloq. 11 (3) (2004), 399-400. 
[2] Argac, N., On prime and semiprime rings with derivations, Algebra colloq. 13 (3) (2006), 371-380.

[3] Beidar, KI, Martindale, WS and Milkhalev, AV, Rings with generalized identities, Marcel Dekker INC 1996.

[4] Bresar, M., Functional identities of degree two, J. Algebra 172 (1995), 690-720.

[5] Maksa, Gy., A remark on symmetric biadditive functions having non-negative diagonalization, Glasnik. Mat. 15 (35) (1980), 279-282.

[6] Maksa, Gy., On the trace of symmetric biderivations, C. R. Math. Rep. Acad. Sci. Canada 9 (1987), 303-307.

[7] Mayne, J. H., Ideals and centralizing mappings of prime rings, Proc. Amer. Math. Soc. 86 (1982), 211-212.

[8] Posner, E.C., Derivations in prime rings, Proc. Amer. Math. Soc. 8 (6) (1957), 1093-1100.

[9] Vukman, J., Symmetric biderivations on prime and semiprime rings, Aequationes Math. 38 (1989), 245-254.

[10] Vukman, J., Two results concerning symmetric biderivations on prime rings, Aequationes Math. 40 (1990), 181-189. 